\section{How Should Canadian Pharmacy Departments Utilize Idarucizumab?}

The direct-acting oral anticoagulants (apixaban, dabigatran, and rivaroxaban) are indicated for prevention of venous thromboembolism in patients undergoing total knee or hip arthroplasty, acute treatment of venous thromboembolism, and prevention of stroke in atrial fibrillation; in all of these situations, they have demonstrated efficacy and safety equivalent or superior to those of traditional anticoagulants. The primary complications of these medications, as shown in clinical trials and observational registries, are major and minor bleeding. ${ }^{1,2}$ The rate of major bleeding in patients treated with direct-acting oral anticoagulants in clinical trials was between $1 \%$ and $3 \%,{ }^{3}$ and $1.8 \%$ of patients enrolled in the Outcomes Registry for Better Informed Treatment of Atrial Fibrillation (ORBIT-AF) experienced major bleeding. ${ }^{2}$ Nonetheless, relative to warfarin, direct-acting oral anticoagulants had statistically significant decreases in the rates of major bleeding ( $4.64 \%$ versus $4 \%)$ and fatal bleeding $(0.52 \%$ versus $0.3 \%){ }^{4}$

Idarucizumab, an antidote for dabigatran, was recently approved for use in Canada. Idarucizumab is a humanized monoclonal antibody fragment that binds both free and fibrin-bound dabigatran. The affinity of dabigatran for idarucizumab is about 350 times greater than its affinity for thrombin. ${ }^{5}$ Within minutes of administration, idarucizumab completely reverses the action of dabigatran, an effect that lasts for up to $24 \mathrm{~h} .{ }^{6} \mathrm{~T}$ hrombosis Canada recommends that idarucizumab be used in severe or lifethreatening bleeding if "dabigatran level $\geq 30-50 \mathrm{ng} / \mathrm{mL}$ or dilute thrombin time ... unavailable and clinically significant dabigatran levels suspected". ${ }^{7}$ Health Canada has approved idarucizumab for use in emergency surgery or urgent procedures and for lifethreatening or uncontrolled bleeding, ${ }^{8}$ which is in accordance with the inclusion criteria for the major clinical trial of idarucizumab, REVERSE-AD. ${ }^{9}$ In the interim analysis of the REVERSE-AD study, ${ }^{9}$ idarucizumab restored hemostasis in a median of $11.4 \mathrm{~h}$. Thirty-three of the 36 patients who required emergency surgery or an invasive procedure had normal intraoperative hemostasis, whereas mild or moderately abnormal hemostasis was seen in only 2 and 1 patients, respectively. ${ }^{10}$ The adverse effects, which appear to be mild, include infusion-site reactions and flushing. ${ }^{6}$ However, 5 patients (6\% of the study population) experienced thrombotic events 2-26 days after administration of idarucizumab, none of whom were receiving antithrombotic therapy when the events occurred. For one of these patients, the thrombotic event was a fatal ischemic stroke 26 days after treatment. ${ }^{9}$ Although data are so far available for only a small number of patients (given that the published study was an interim analysis), this signal highlights the importance of reassessing the benefits and risks of antithrombotic therapy after management of the acute bleeding event.

Idarucizumab has a standardized 5-g IV dose, which is administered as two $2.5-\mathrm{g}(50-\mathrm{mL})$ bolus infusions over no longer than 5-10 min and no more than $15 \mathrm{~min}$ apart. No reconstitution is required before administration, but idarucizumab requires refrigeration during storage. ${ }^{10}$ The cost per treatment is $\$ 3750$; however, direct costs to institutions may vary. ${ }^{11}$ By comparison, digoxin immune $\mathrm{FAB}$, an antibody used in the treatment of digoxin toxicity, is $\$ 858.71$ per 40-mg vial, ${ }^{12}$ with a $70-\mathrm{kg}$ patient possibly needing up to 14 vials, depending on serum digoxin concentration. ${ }^{13}$

Aripazine (also known as PER977 or ciraparantag), a small synthetic molecule with potential as a universal anticoagulant reversal agent, and andexanet alfa, a modified recombinant factor Xa molecule that reverses oral and injectable factor Xa inhibitors (e.g., apixaban, rivaroxaban, enoxaparin, fondaparinux), are currently undergoing testing but have not yet been submitted to Health Canada for approval. ${ }^{10}$ The ANNEXA-A and ANNEXA-R trials evaluated the efficacy and safety of andexanet alfa in healthy, older volunteers receiving either apixaban $5 \mathrm{mg}$ twice daily or rivaroxaban $20 \mathrm{mg}$ daily. Andexanet alfa reversed anticoagulation within minutes after 
administration without evidence of thrombotic events or serious adverse events. ${ }^{14}$ Currently underway is a study of andexanet alfa in patients with major bleeding who are receiving direct and indirect oral anticoagulants. ${ }^{15}$

Although the introduction of target-specific antithrombotic reversal agents is a significant advance in the management of major bleeding in patients receiving direct-acting oral anticoagulants, supportive measures should still be used in patients who are receiving anticoagulation therapy and who present with major bleeding. Given the Thrombosis Canada recommendations, which include the use of idarucizumab for patients who present with severe or life-threatening bleeding while taking dabigatran, we recommend that health care organizations review this agent in an expedient manner to determine whether it fits within their respective bleeding protocols and, if appropriate, add it to the formulary. Such formulary decisions should include consideration of the fact that the published REVERSE-AD trial was an interim analysis involving the first 90 of a planned 300 patients. ${ }^{10}$ To ensure proper utilization, we also recommend development of specific criteria for use through a multidisciplinary approach, including consultation with emergency physicians, trauma surgeons, critical care physicians, anesthesiologists, hematologists, and other vested stakeholders. A quality assurance system should be developed to ensure continued correct utilization of this agent. Given the high cost of idarucizumab, hospitals in geographic proximity might consider sharing a central supply. Finally, the importance of restarting anticoagulation once it is safe to do so cannot be overlooked and should be impressed upon those involved in the patient's care.

\section{References}

1. Crowther MA, Warkentin TE. Bleeding risk and the management of bleeding complications in patients undergoing anticoagulant therapy: focus on new anticoagulant agents. Blood. 2008;111(10):4871-9.

2. Steinberg BA, Kim S, Piccini JP, Fonarow GC, Lopes RD, Thomas L, et al. Use and associated risks of concomitant aspirin therapy with oral anticoagulation in patients with atrial fibrillation: insights from the outcomes registry for better informed treatment of atrial fibrillation (ORBIT-AF) registry. Circulation. 2013;128(7):721-8.

3. Liew A, Eikelboom JW, O’Donnell M, Hart RG. Assessment of anticoagulation intensity and management of bleeding with old and new oral anticoagulants. Can J Cardiol. 2013;29(7 Suppl):S34-S44.

4. Chai-Adisaksopha C, Crowther M, Isayama T, Lim W. The impact of bleeding complications in patients receiving target-specific oral anticoagulants: a systematic review and meta-analysis. Blood. 2014;124(15):2450-8.

5. Schiele F, van Ryn J, Canada K, Newsome C, Sepulveda E, Park J, et al. A specific antidote for dabigatran: functional and structural characterization. Blood. 2013;121(18):3554-62.

6. Glund S, Stangier J, Schmohl M, Gansser D, Norris S, van Ryn J, et al. Safety, tolerability, and efficacy of idarucizumab for the reversal of the anticoagulant effect of dabigatran in healthy male volunteers: a randomised, placebo-controlled, double-blind phase 1 trial. Lancet. 2015; 386(9994):680-90.
7. Clinical guides: new/novel oral anticoagulants (NOACs): management of bleeding. Hamilton (ON): Thrombosis Canada; [updated 2016 Jan 12; cited 2016 Sep10]. Available from: http://thrombosiscanada.ca/ ?page_id=18\#

8. Praxbind ${ }^{\mathrm{TM}}$ [product monograph]. Boehringer Ingelheim Pharma GmbH \& Co KG; 2016 Apr 29 [cited 2016 May 2]. Available from: www. hc-sc.gc.ca/dhp-mps/prodpharma/databasdon/index-eng.php

9. Pollack CV Jr, Reilly PA, Eikelboom J, Glund S, Verhamme P, Bernstein $\mathrm{R}$, et al. Idarucizumab for dabigatran reversal. $N$ Engl J Med. 2015; 373(6):511-20.

10. Antidote treatments for the reversal of direct oral anticoagulants. Ottawa (ON): Canadian Agency for Drugs and Technology in Health; 2015 June 23 [cited 2015 Dec 16]. Available from: www.cadth.ca/antidotetreatments-reversal-direct-oral-anticoagulants

11. Praxbind. In: FormularyKits.ca real-time payer portal [database]. Mississauga (ON): FormularyKits.ca; [cited 2016 May 8]. Available from: https://formularykits.ca. Login required to access content.

12. DigiFab ${ }^{\mathrm{TM}}$ digoxin immune fab (ovine). In: Pharmaclik [online order management system]. McKesson Canada; [cited 2015 Dec 16]. Available from: https://clients.mckesson.ca/auth/Login?Template=login_template \&GAREASONCODE =-1 \&GARESOURCEID =USRLOG \& GAURI=https://clients.mckesson.ca/logon\&Reason=-1\&APPID=USRLOG\&URI=https://clients.mckesson.ca/logon. Login required to access content.

13. DigiFab (digoxin immune fab ovine) [product monograph]. Savage Laboratories; 2002 [cited 2015 Dec 16]. Available from: www.savagelabs.com/Products/DigiFab/Monograph/DigiFab\%20Monograph.pdf

14. Siegal DM, Curnutte JT, Connolly SJ, Lu G, Conley PB, Weins BL, et al. Andexanet alfa for the reversal of factor Xa inhibitor activity. $N$ Engl J Med. 2015;373(25):2413-24.

15. Portola Pharmaceuticals. A study in patients with acute major bleeding to evaluate the ability of andexanet alfa to reverse the anticoagulation effect of direct and indirect oral anticoagulants [clinical trial registration]. In: ClinicalTrials.gov. Bethesda (MD): US National Institutes of Health; [record verified $2015 \mathrm{Oct}$; cited 2015 Dec 16]. Available from: https: //clinicaltrials.gov/ct2/show/NCT02329327?term=andexanet+alfa\&rank=3

\author{
Kirsten Tangedal, BSP, ACPR \\ Staff Pharmacist \\ William Semchuk, MSc, PharmD, FCSHP \\ Manager Clinical Pharmacy Services \\ Jennifer Bolt, BSCPharm, ACPR, PharmD \\ Manager of Research and Development \\ Department of Pharmacy Services \\ Regina Qu'Appelle Health Region \\ Regina, Saskatchewan
}

Competing interests: William Semchuk has received speaker's honoraria and Jennifer Bolt has received personal fees from Boehringer Ingelheim, for activities unrelated to this submission. No other competing interests were declared. 